\title{
An Interview with James Medcalf: CAMCOG
}

\author{
Michael F Shaughnessy* \\ Department of Neurology, USA
}

*Corresponding author: Michael F Shaughnessy, Department of Neurology, USA.

Keywords: CAMCOG (A Useful Screening Instrument for Dementia in Stroke Patients)

First of all, can you tell us about yourself and your education and experience?

I hold a degree in Biotech from the University of Nottingham and I have been with Cambridge Cognition for 9 years, working with Academia, various pharmaceutical markets and various healthcare markets.

Now, can you tell us about CAMCOG- How it was developed, who developed it and why?

The test was originally developed as the world's first computerized touchscreen conative tests over 30 years ago by Prof Barbara Sahakian and Prof Trevor Robins. As Cambridge Cognition we developed in 2001 as our own company to commercialize these tests. Since then the tests have been used in over 2300 published papers and 260 clinical trials and two medical devices (EMA, TGA and FDA have been approved) We have a platform for clinical trials and academic research as well as several medical devices.

How is it administered and what kind of qualifications do you need?

The system administers the tests for you by speaking the instructive instructions in any one of 47 languages. Thus, no qualifications are needed to administer the tests. We have both in lab/clinic platform which is administered and a web-based version for tests that do not require strict device timings. For the in-lab version, the test administrator has to logon, add or select the subject and press start tests. After that the system does the administration for you. On the web based, the subjects are not supervised at all. The system goes though some checks, like sound check and then does the tests.

Let's talk about subtest- does it assess reaction time, and if so how?

Table 1:

\begin{tabular}{|c|c|c|c|c|c|}
\hline Test Name & Short & Adult Normative Data & Web Based Testing & Tone Mode & Adv Admin Time Min \\
\hline Pattern Recognition Memory & PRM & Yes & Yes & No & 4 \\
\hline One Touch Stockings of Cambridge & OTS & Yes & Yes & No & \multicolumn{2}{|c|}{ No } \\
\hline Emotional Recognition Task & ERT & Yes & Yes & Yes & 4 \\
\hline Paired Associate Learning & PAL & Yes & Yes & 7 \\
\hline Spatial Working Memory & SWM & Yes & Yes & Yes & \\
\hline $\begin{array}{c}\text { Rapid Visual Information } \\
\text { Processing }\end{array}$ & RVP & Yes & Yes & No & \\
\hline Spatial Span & SSP & Yes & Yes & Yes & 7 \\
\hline Emotional Bias Test & EBT & Yes & Yes & \\
\hline Intra/Extra Dimensional Set Shift & IED & & Yes & &
\end{tabular}




\begin{tabular}{|c|c|c|c|c|c|}
\hline Delayed Match to Sample & DMS & No & Yes & No & 7 \\
\hline Cambridge Gambolling Task & CGT & No & Yes & Yes & 18 \\
\hline Stockings of Cambridge & SOC & No & Yes & Yes & No \\
\hline Motor Screening Task & MOT & No & No & No & 8 \\
\hline Multi-Tasking Test & MTT & No & No & No & 15 \\
\hline Stop Signal Task & SST & No & No & Yes & 3 \\
\hline Verbal Recognition Memory & VRM & No & No & No & 5 \\
\hline Reaction Time Test & RTI & No & No & \\
\hline
\end{tabular}

Each CANTAB test is designed to measures specific cognitive domains. This way you can focus your testing time on areas of cognition important to your projects (Table 1 ).

This is for the standard modes. We also have higher functioning modes for healthy young subjects.

\section{Is memory evaluated and are we talking short term, long term or working memory?}

We have a suite of different memory tests and would aim to match your research goals to the right tests

for you. PAL - this is a visual Episodic memory test SWM is a working memory test. Spatial Span is a visual analogue of the digit span with both short- and longer-term parts to it VRM is a word list type test with short and longer term parts to it.

\section{Executive functions- how is this evaluated?}

We have several executive functioning tests: SOC and OTS are analogues of the tower of Hanoi type tests. SWM has an executive functioning outcome based upon how the subject performs on the test.

\section{What is the CANTAB Emotion Recognition Task and} why is this important?

The ERT was developed by Bristol University as a repeatable emotional recognition type test to replace the classical Ekman faces test. The ERT uses 6 key emotions. With each emotion graded from $0 \%$ (neutral) to $100 \%$ in 15 stages. This was achieved by using a generated face morphed. The face is shown for $200 \mathrm{~ms}$ (subconscious emotional recognition) followed by an image of static for $250 \mathrm{~ms}$ (removed the face image from the retina) and then asked to choose the emotion shown (forced choice)
Psychomotor speed- are we talking about reaction time here? Or auditory or visual reaction?

The reaction Time test has a button on the bottom of the screen and 5 (or 1 depending on the mode) circles at the top. The subject presses and holds down the button. A yellow spot will appear in one of the circles. The subject then needs to release their finger and using the same finger press the circle where the yellow spot appeared.

The reaction time Is measured from when the yellow spot appears to when the subject releases their finger. Movement speed is calculated from the time from release to when they press the circle. This test requires strict timings on the device, especially around the devices error in measurement rates. This is why this test is only validated for us on the iPad, which passed our inhouse robotics testing. Android tables all failed, the cheaper ones appalling so.

I understand there is some sort of self-report questionnaire- why is this included? What does it assess?

We do provide eCOA questionnaires for clinical trials. This is because we have a platform that is fully regulatory compliant for clinical trials, thus adding questionnaires to this made sense to us.

Is there a web site where interested clinicians can gain more information?

https://www.cambridgecognition.com

Acknowledgement

None.

\section{Conflict of Interest}

No conflict of interest. 